\title{
Highly integrated magnetoresistive sensors in aerospace applications
}

\author{
Dr. Rolf Slatter, CEO \\ Sensitec $\mathrm{GmbH}$ \\ Lahnau, Germany \\ rolf.slatter@sensitec.com
}

\begin{abstract}
The usage of magnetic sensors is increasing steadily in the field of electric drives. Above all magnetoresistive (MR) sensors are experiencing a significant increase in applications in motion control and power electronics systems, not only in the industrial and automotive fields, but also in aerospace applications.

MR sensors are not only used for measuring rotational and linear motion, but also for non-contact switching applications and furthermore for highly dynamic current measurement. This is largely the result of increasingly complex demands on the sensors for high performance electric drives [1]. Sensors must not only be accurate and dynamic, but must also be robust under difficult operating conditions and exhibit very high reliability. Recent developments, such as the trend to electro-mobility, are generating additional demands, with respect to compact dimensions and energy efficient operation. This combination of demands is leading to the more intensive use of magnetic sensor principles, compared to optical, inductive or capacitive principles.
\end{abstract}

Keywords: aerospace sensors, current sensor, magnetic sensor, electrical environmental control system, position pick-off unit

\section{Introduction}

The magnetoresistive effect is best known from the read heads of computer hard discs or from magnetic memory (MRAM) applications, but it is also well suited to uses in sensor technology. It has a long history, the anisotropic magnetoresistive (AMR) effect being first discovered in 1857 by Lord Kelvin. The AMR effect occurs in ferromagnetic materials, such as nickel-iron layers structured as strip elements, whose specific impedance changes with the direction of an applied magnetic field. Due to a special structure of the strips the resistance change is proportional to the applied magnetic field over a wide range. This means that by adept design of the sensor structure very small magnetic fields can be detected with very high accuracy.

However, the MR-effect did not experience widespread use until the early 1980s, when the first MR-based read heads were implemented in hard disc drives. The first industrial applications for MR-based sensors followed at the beginning of the 1990s, since when the number of applications has increased dramatically. The applications are not only limited to terrestrial use - MR sensors are used to control the electric drives used on "Curiosity", the Planetary Rover that landed successfully on Mars in August 2012. MR sensors are also used extensively in safety-critical automotive applications, for example in wheel speed sensors for the ABS (anti-lock braking) system or in steering angle sensors for the ESP (electronic stability program) system.

The small dimensions, large air gap and high accuracy make MR sensors a good replacement for resolvers or synchros in angle and speed measurement applications.

The magnetoresistive effect is also particularly attractive in the field of electrical current measurement. The very high sensitivity means that there is no need to use an iron core to concentrate the magnetic field generated by the conductor carrying the current. This means that MR-based current sensors do not suffer from hysteresis and that they have a significantly higher bandwidth. Compared to shunt resistors MR-based sensors have the benefit of galvanic isolation and dramatically lower power losses. This is particularly important in high voltage applications and where overall power efficiency is a major design driver, as the case of "More Electric Aircraft (MEA)" applications. 


\section{Highly integrated current sensors}

Sensitec has a long experience of developing MR-based current sensors for industrial applications. The increased demand for very compact current sensors generated by the recent trend to electro-mobility was the driver for the development of a highly integrated current sensor (CFS1000) comprising an AMR sensor chip, a signal conditioning ASIC (application-specific integrated circuit) and two biasing permanent magnets (Fig. 1) [2].

The latter are necessary for maintaining the initial magnetization direction of the AMR structures in the case of overcurrent situations. The permanent magnet material and the AMRsensitive sensor material are applied onto wafer substrates by a special process and thus can be processed further with standard semiconductor methods, concerning singularization or assembly.

A special lead-frame as well as an advanced assembly technique enable a "system-inpackage" solution (SIP): all system components are molded within a JEDEC compliant SOIC16 package (Fig. 1). The product can be mounted with standard pick-and-place equipment onto a PCB and subsequently reflow soldered.

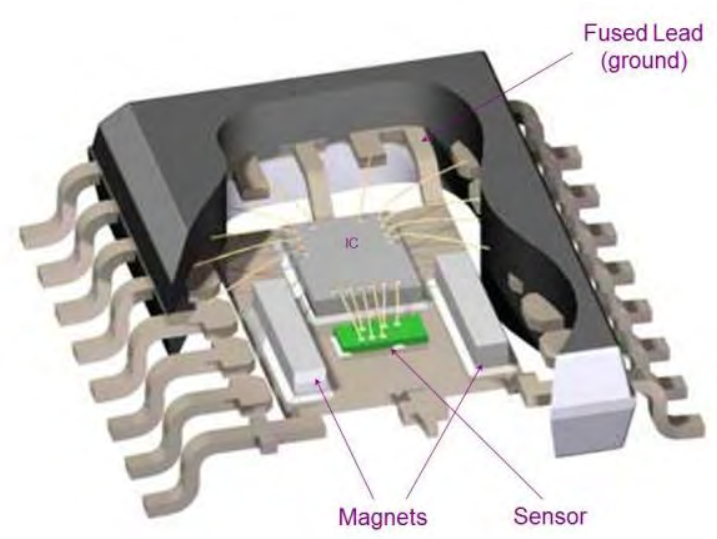

Fig. 1. CFS1000 Current Sensor

The quantity to be measured is a differential magnetic field, also referred to as field gradient, that is generated by two currents with opposed current flow directions. The primary current conductor is typically U-shaped, with its straight parallel parts positioned underneath the sensor (Fig. 2).

For current measurement four AMR "resistors" are connected to form a Wheatstone Bridge. The resistors on the silicon chip are placed so that they constitute a differential field sensor. This is necessary because interference fields can be eliminated this way. Combined with a signal conditioning and processing ASIC the chip is assembled on a substrate, which can be a hybrid circuit or a lead frame (Fig. 3).

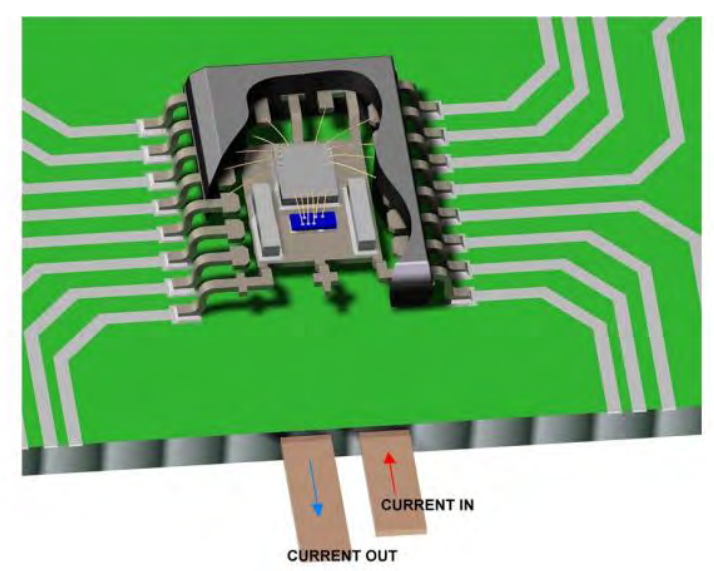

Fig. 2. U-shaped conductor embedded in PCB

Furthermore, a compensation conductor is integrated on the chip with which a magnetic field can be generated close to the resistors. On the opposite side of the substrate the primary current conductor is led in a U-shape below the MR chip.

The geometry of the primary conductor defines the measurement range of the current sensor. Based on the output signal from the MR chip, the ASIC (shown in Fig. 3 schematically as an operational amplifier) generates a current $I_{\text {comp }}$ in the compensation conductor, which compensates the magnetic field generated by the primary conductor in the plane of the AMR resistors. With this method the signal achieves a high linearity $(0.1 \%)$ and is largely independent of temperature. This compensation current is directly proportional to the primary current to be measured and is used to generate the output signal from the current sensor.

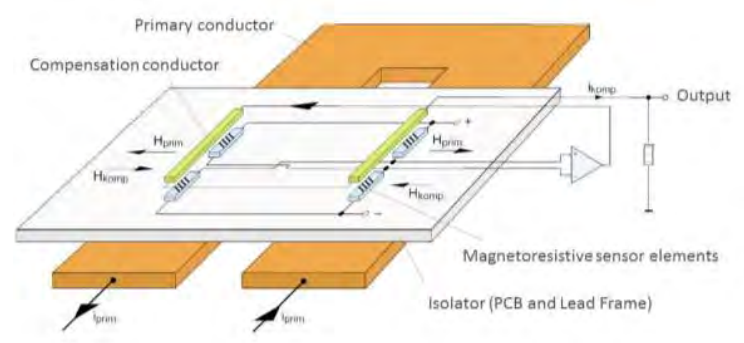

Fig. 3. Principle of Operation

This "closed-loop" principle results in an extremely compact sensor that is largely insensitive to homogeneous interference fields and temperature changes, with a low power 
consumption and very high efficiency. The AMR-based current sensor exhibits no hysteresis as observed in iron core based Hallsensor solutions and no remaining magnetic offset after overcurrent events. Due to the high sensitivity of the AMR sensor chip, a flux concentrator is not necessary.

The sensor is designed for high accuracy and very fast electronic measurement from $\mathrm{DC}$ up to $500 \mathrm{kHz}$ AC. Contrary to Hall-effect based sensors, the described system enables differential magnetic field measurement by means of an advanced geometry of the magnetoresistive elements. Due to this construction the sensor is immune to homogeneous interference fields and hence needs no magnetic shielding as required for surface mounted Hall-effect current sensors with external current bar.

By variation of the geometry of the external primary current bar, the CFS1000 can be adapted to different current ranges and applications. This means that only one sensor type is needed for a wide range of applications for rated currents from 10 to more than $1000 \mathrm{~A}$ (Fig. 4). The system accuracy can be improved by adjusting both offset and sensitivity in the target application of the customer and by using either the internal or an external reference voltage. The adjustable over-current detection enables fast response in overload situations to prevent damage to the power transistors.

A more detailed description of the principle of operation and the advantages of the CFS 1000 can be found in [3].

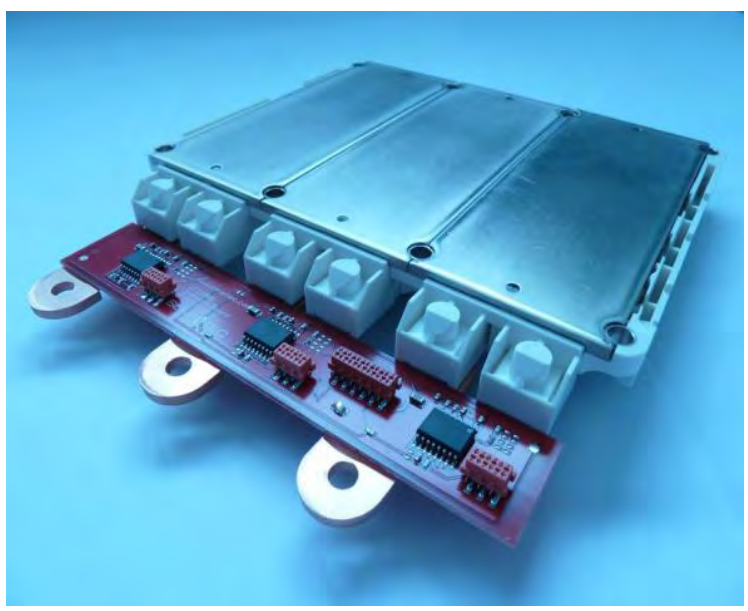

Fig. 4. 3-phase module using CFS1000 current sensors

\section{Performance Tests}

The key specifications of the CFS1000 current sensor are listed in Table 1.

Tab. 1: Key specifications for CFS1000 current sensor

\begin{tabular}{|l|l|l|l|l|}
\hline \multicolumn{1}{|c|}{ Parameter } & Min. & \multicolumn{1}{c|}{ Typ. } & Max. & Unit \\
\hline Supply voltage & 4.75 & 5.00 & 5.25 & $\mathrm{~V}$ \\
\hline $\begin{array}{l}\text { Primary nominal current } \\
(\mathrm{RMS})^{1)}\end{array}$ & 10 & - & 1000 & $\mathrm{~A}$ \\
\hline $\begin{array}{l}\text { Primary measuring range } \\
(\text { abs })^{2)}\end{array}$ & 30 & - & 3000 & $\mathrm{~A}$ \\
\hline $\begin{array}{l}\text { Nominal output current } \\
(\mathrm{RMS})\end{array}$ & - & 2 & - & $\mathrm{mA}$ \\
\hline $\begin{array}{l}\text { Upper cut-off frequency } \\
(-3 \mathrm{~dB})\end{array}$ & - & 500 & - & $\mathrm{kHz}$ \\
\hline Overall accuracy ${ }^{3)}\left(\mathrm{T}=25^{\circ} \mathrm{C}\right)$ & - & - & \pm 1 & $\%$ \\
\hline $\begin{array}{l}\text { Overall accuracy } \\
\left(\mathrm{T}=-40^{\circ} \mathrm{C} \text { bis }+125^{\circ} \mathrm{C}\right)\end{array}$ & - & - & \pm 2 & $\%$ \\
\hline Temperature range & -40 & - & +125 & ${ }^{\circ} \mathrm{C}$ \\
\hline
\end{tabular}

1) The measuring range is defined by the geometry of the external primary current bar.

2) Restricted to $1 \mathrm{~s}$ in a 60 s interval.

3) The overall accuracy includes offset, linearity and sensitivity error $(\varepsilon \Sigma=\varepsilon G+\varepsilon$ off $+\varepsilon$ lin $)$.

Extensive tests have been undertaken as part of the AEC-Q100 qualification for automotive applications. Fig. 5 shows the frequency response of the current sensor. It shows that the $-3 \mathrm{~dB}$ cut-off-frequency is well in excess of $500 \mathrm{kHz}$. There is no frequency derating as experienced with hall-based sensors (at lower frequencies than this).

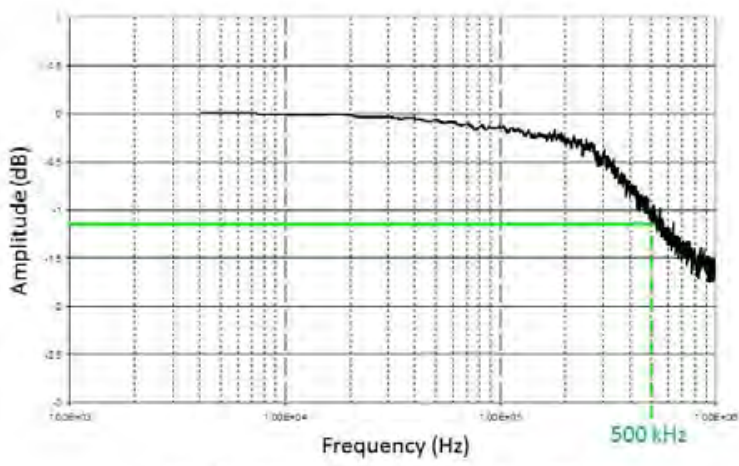

$3 \mathrm{~dB}$ corner frequency: $>500 \mathrm{kHz}$

Fig. 5. Frequency response

Fig. 6 shows the step response of the current sensor. The response time is in the range of $600 \mathrm{~ns}$, which is an exceptional value. This fast response is very effective in protecting power transistors in the event of short-circuits. 


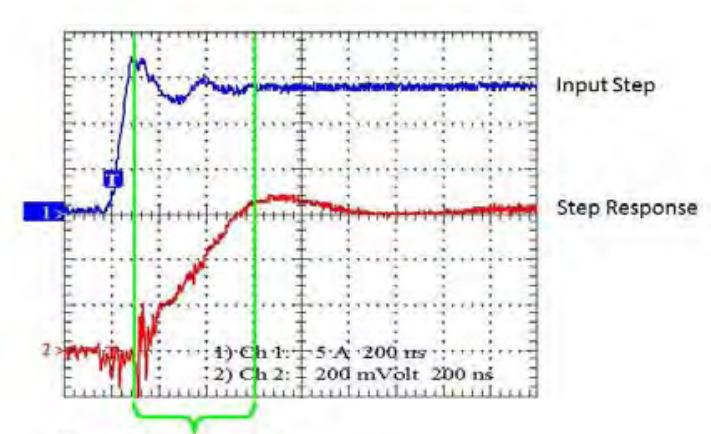

Rise Time $<600$ ns

\section{Fig. 6. Step response}

\section{Application Example}

Following on from the POA (Power Optimised Aircraft) and More Open Electrical Technologies (MOET) initiatives, the European Union is now part-funding the Cleansky Initiative, where one of the main activities is the replacement or enhancement of hydraulic and pneumatic systems by means of electrically driven actuators, supported by the latest sensor technology.

The focus is on the electrification of high-lift systems, the landing gear and the environmental control system. MR sensors have been applied successfully in demonstrators in all three areas for either angle sensing or current sensing applications [4]. In this paper the application of highly integrated current sensors in an electrical environmental control system (E-ECS) will be described.

At aircraft level, the electrification of the ECS is a key enabler of the More Electric Aircraft, as it is the biggest steady state power consumer during aircraft cruise. An electrical ECS could also enable the saving of a lot of fuel, due to its consequential adaptability and a reduced impact on the operation of the engine [5], [6].

The task of an Environmental Control System (ECS) is to control air temperature, to pressurize relevant aircraft compartments, to provide sufficient ventilation and fresh air to passengers, to control the level of humidity within acceptable limits in the cabin, and to remove pollutants. A conventional ECS uses air bled from the engine compressor to provide conditioned air to the cabin.

The numerous benefits of an electrical ECS include improved engine design, better fuel efficiency and improved functionality, because the E-ECS can be operated independently from the operational cycles of the engine [7].

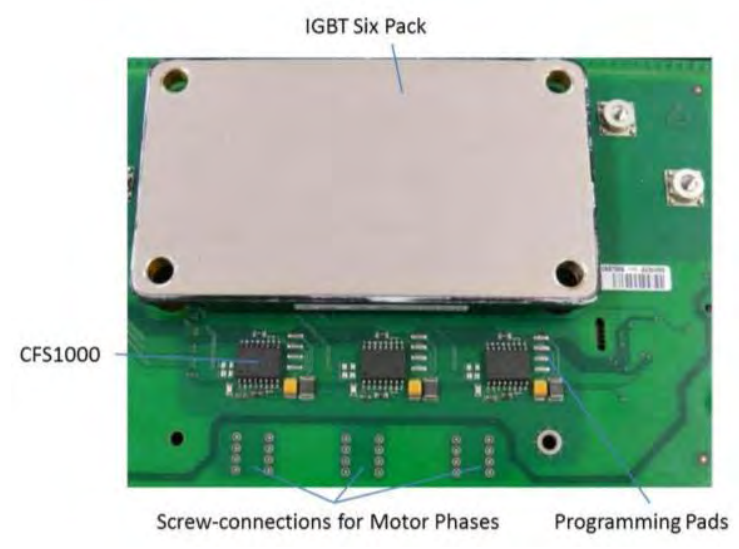

Fig. 7. PCB from Electrical Environmental Control System

(Source: Liebherr Elektronik $\mathrm{GmbH}$ )

Within the Cleansky project a German aerospace supplier has the task of optimizing the E-ECS. The target is to increase the power density of the power electronics, compared to previous solutions, by a factor of 4 . CFS 1000 current sensors have been selected for the following reasons:

- Extremely small size to reduce volume and weight of the complete power electronics

- High shock and vibration resistance of the automotive-qualified product

- High accuracy to allow more accurate dimensioning (and therefore downsizing) of other electronic components

The AMR current sensors are used to measure rated current of up to $65 \mathrm{~A}$ passing through a conductor embedded in the power electronics PCB (Fig. 7). The current sensors are mounted between the IGBT power pack and the threephase motor.

The application of MR-based current sensors has enabled an extremely compact design of the power electronics for the E-ECS.

\section{Integrated Angle and Speed Sensors}

Angle and speed measurement are traditionally two of the main application areas for MR sensors. A wide variety of well-known electric motors and encoders are now equipped with MR sensors to provide feedback for the control system.

The main reasons for using MR sensors are:

- High accuracy and repeatability

- Fast dynamic response

- High robustness 
- High reliability

- Small dimensions

- Low power consumption

- Contactless measurement principle
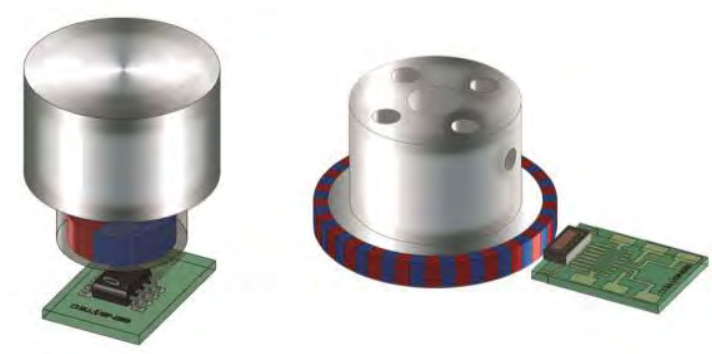

Fig. 8. Angle measurement at shaft end (left) and shaft circumference (right)

Based on these benefits MR sensors have been a significant enabling technology for the most recent motor and encoder generation with much improved power density, improved power efficiency and improved reliability.

Figure 8 shows typical sensor configurations for on-axis (shaft end) and off-axis (Shaft circumference) angle measurement. Typically a disc- or ring shaped magnet is used as target and the MR sensor and signal processing electronics are mounted on a common PCB.

\section{Application Example}

Within a national aerospace research project magnetoresistive sensors have been applied in a new concept for a position pick-off unit (PPU) as part of an improved high-lift system for passenger aircraft. The PPU is used to measure the position of the slats and flaps to ensure that there is no asymmetry between the port and starboard wings [8],[9].

The slats and flaps are driven by hydraulic actuators mounted at the wing root. Power is transmitted to the slats and flaps by means of rotating shafts and gearboxes mounted behind the leading edge of the wing. PPUs are installed near to the wing root and wing tip.

Typically the PPU incorporates a high reduction ratio gear to reduce a number of revolutions of the rotating shaft to an angle of less than $360^{\circ}$, in order to enable a resolver to give an absolute angular output signal.

In the above-mentioned project Airbus, Harmonic Drive AG and Sensitec have co- operated in order to replace the traditional resolver with a compact MR sensor.

Figure 9 shows a section through the new MRPPU. MR sensors are used to measure both angle, as well as speed.

The high-speed input shaft of the PPU has a spur gear profile, which is the measurement target for the MR speed sensor.

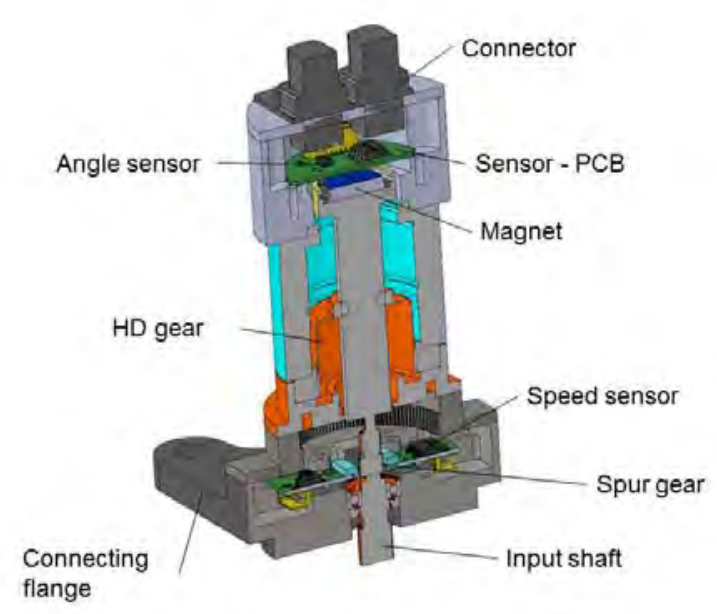

Fig. 9. MR-PPU: Position Pick-Off Unit with magnetoresistive sensors (Source: Harmonic Drive AG)

The speed and angular displacement of the input shaft is reduced by a two-stage Harmonic Drive (HD) gear. A magnet attached to the lowspeed output shaft of the HD gear is used to generate an absolute angle signal via the MR angle sensor.

Both sensors are provided with digital signal processing electronics to generate digital speed and angle information within the MR-PPU.

The new PPU design offers a number of advantages compared to previous, resolverbased, designs:

- Higher absolute angular accuracy $\left(0.17^{\circ}\right.$ compared to $\left.0.3^{\circ}\right)$

- Smaller dimensions

- Significantly lower weight $(<500 \mathrm{~g})$

- Additional functionality (integrated speed measurement)

- Digital rather than analogue data transfer

- Reduced computational load for slat and flap control computer (SFCC) 


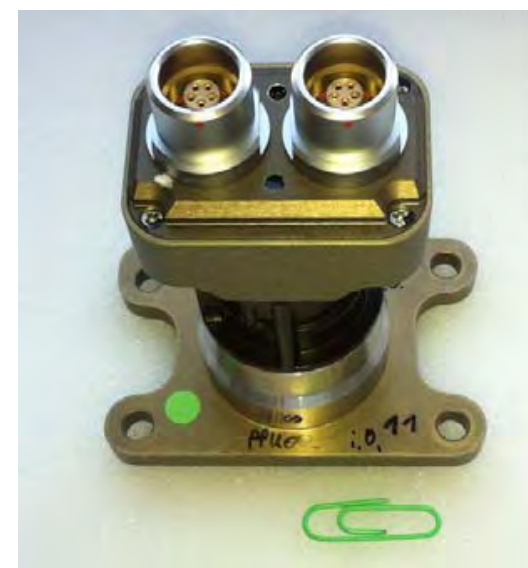

Fig. 10. Photo of MR-PPU prototype (Source: Harmonic Drive AG)

A prototype MR-PPU is currently undergoing performance and environmental tests at the time of writing.

\section{Outlook}

Magnetoresistive sensors are opening up new possibilities for the designers of electrical equipment for aircraft. The combination of small size, high precision, high bandwidth and robustness make MR sensors very attractive for the measurement not only of electrical current, but also of angle, position and length.

Following on from the success of MR sensors in numerous space applications, for example, for monitoring almost all moving mechanisms on the Mars Rover "Curiosity", it can be expected that in the near future MR sensors will be used in a growing variety of aircraft applications as well [10].

\section{References}

[1] R. Slatter, „Magnetoresistive Sensors for HighPerformance Electric Drives", Proc. of Electric Drives Production Conference, Nürnberg, 2012

[2] J. Bockstette et al, "Bidirectional current controller for combination of different energy systems in HEV / EV", Proc. of Power Electronics, Machines and Drives Conference, Bristol, 2012

[3] S. Scherner et al, "Integrated Current Sensor based on Magnetoresistive (MR) Technology, Proc. of PCIM Conference, Nürnberg, 2012

[4] I. Schäfer, "Aviation Sensor Requirements: Do they fit to MR Technology?", Proc. of 11th MR Symposium, Wetzlar, 2011

[5] L. Faleiro et al, "Integrated Equipment Systems for a More Electric Aircraft - Hydraulics and Pneumatics", Proc. of 24th International Congress of the Aeronautical Sciences, 2004

[6] A. A. AbdElhafez \& A. J. Forsyth, „A Review of More Electric Aircraft", Proc. of 13th International
Conference on Aerospace Science and Aviation Technology, Cairo, 2009

[7] G. Galzin et al, "Electrical Environmental Control System", Proc. of More Electric Aircraft Forum, 2009

[8] I. Schäfer, "Doppelte Funktion - Sichere Flugzeuge durch hochwertige Getriebe", Antriebstechnik 10/2007

[9] M. Recksiek, Advanced High Lift System Architecture with Distributed Electrical Flap Actuation, Proc. of AST - Workshop on Aviation System Technology, Hamburg, 2009

[10] M. Hartmann, "Ultra-Compact and Ultra-Efficient Three-Phase PWM Rectifier Systems for More Electric Aircraft", Diss. ETH No. 19755, ETH Zürich, 2011 